\title{
Computer-Aided Color Aesthetic Evaluation Method Based on the Combination of Form and Color
}

\author{
Wenke Kang, ${ }^{1}$ Shengfeng Qin, ${ }^{2}$ and Quan Zhang ${ }^{3}$ \\ ${ }^{1}$ School of Industrial Design, Northwestern Polytechnical University, Xian 710072, China \\ ${ }^{2}$ School of Design, Northumbria University, Newcastle upon Tyne NE1 2SW, UK \\ ${ }^{3}$ Parametric Technology Software Co., Ltd., Shanghai 2000202, China \\ Correspondence should be addressed to Wenke Kang; kangwenkekkk@nwpu.edu.cn
}

Received 3 June 2014; Accepted 19 December 2014

Academic Editor: Carsten Proppe

Copyright (C) 2015 Wenke Kang et al. This is an open access article distributed under the Creative Commons Attribution License, which permits unrestricted use, distribution, and reproduction in any medium, provided the original work is properly cited.

\begin{abstract}
This paper presents a new method of color aesthetic evaluation based on the combination of form and color. According to the human visual physiological and psychological characteristics, this paper first proposes a new form-color field theory for the coupled formcolor aesthetic evaluation based on the psychophysical field theory and the Moon and Spencer model. Second, it builds a coupled form-color topological graph for describing their interaction and develops a strength calculation algorithm for color harmony based on the new form-color field theory. Finally, it develops an aesthetic measure evaluation model for coupled form-color fields to evaluate the new theory. The experiment results show that the new coupled form-color aesthetic evaluation method is useful and can be integrated into an intelligent evaluation process for color design scheme.
\end{abstract}

\section{Introduction}

Nowadays, the functional aspects of many consumer products used in daily life are fully matured. For products with similar functions, their visual images become an important factor in a consumer's shopping decision-making process when deciding which particular product to purchase. The overall image perception of a product's style is generally induced by its form and color. The coupled form-color perception on a product plays a crucial role in today's increasingly competitive marketplace. Therefore, having a desirable product style is very important for product design, and in turn having aesthetic evaluations on product styles is very necessary in a product design process.

In order to evaluate the aesthetic of each form and color, many researchers have studied a lot of examples to analyze what kind of form and color is beautiful. Sato and Oda [1] studied the importance of color and shape to aesthetic evaluation by experiments. The analysis indicated that the aesthetic evaluation of colored shape was influenced by both the color and shape components and the shape component had stronger influence on that of the colored shape though color has the first impressions in the aesthetic evaluation of product design. At the same time, color harmony is one of the most important indicators in the aesthetic evaluation of color.

Aesthetic measure of form and color design has enormous significance in art and design. While the basic mechanisms of color perception have been extensively studied, the semantic and aesthetic aspects of color are still poorly understood. There is a long trail of research into color aesthetic [2-5]. Ou et al. [6] tested the relationship between color harmony and single-color associations and colorcombination association by color experiment and established a model of color association and harmony. But the models of color associations and harmony lack consideration of other design elements, such as shape, texture, and product functionality; therefore, it may only apply to specific layout designs or specific industry sectors. In a later study [7], the same authors studied the rule of the three factors of hue $(H)$, chroma $(C)$, and lightness $(L)$ of color effect on the color harmony. However, the model was developed using uniform color patches placed side by side and the test data set was also based on uniform color patches with a medium gray 
background. Therefore, this model may be not applicable to all possible color combination types, shapes, textures, and background colors. Furthermore, this model is limited to combinations of two colors.

Tsai and Chou [8] used a 3D model of the flask rendered with arbitrary colors by adjusting the RGB parameter values at random to study and develop an automatic design support method based on the principles of aesthetic measurement, gray theory, and genetic algorithms, respectively, and to generate and evaluate color-design candidates. However, the design support system is limited to two-color designs and a single-color association scale. In another study of computeraided product color design with artificial intelligence [9], Tsai et al. established the relationship between multicolored products and their images and introduced a method using gray theory, aesthetic measurement, and a genetic algorithm to generate and evaluate color-design candidates automatically. However, this study did not cover the color harmony affected by the coupling of form and color.

Kimura et al. [10] have investigated the effect of the similarity of affective meanings on harmony between shape and color, and they have revealed the factor determining the harmony.

Form and color appear always together in product design and both of them have the important influence in color aesthetic evaluation; therefore, this paper will focus on the study of the calculation method of aesthetic measure based on the coupling of color and form. The main contributions of this paper are as follows: (1) proposing the aesthetic evaluation method and theory for a multi-color-form coupled field; (2) developing a form-color topological graph based on morphology topology to represent their relationships and realizing the visual expression and calculation of form and color; (3) achieving the harmony intensity evaluation of a coupled form-color field ranging from being qualitative to being quantitative based on the theory of psychophysical field. Its flowchart for color aesthetic measure evaluation is shown in Figure 1.

\section{The Theoretical Foundation of Coupling Form and Color Aesthetic Evaluation Together}

2.1. The Color Aesthetic Measure Theory. G. Birkhoff has given the principle of unity-in-multiplicity, a definite quantitative formulation. The aesthetic measure $M$ of an object may be defined by the general equation

$$
M=\frac{O}{C}
$$

Accordingly, the aesthetic measurement method proposed by Moon and Spencer (MS) [11] is used to evaluate the overall color harmony image of a product rendered with two different colors. This measure is defined as follows:

$$
M=\frac{\sum_{i=1}^{n} O_{i}}{C_{m}+C_{h}+C_{v}+C_{c}},
$$

in which $M$ is the aesthetic measure of a color, $O$ is the number of orders, and $C$ is the complexity. Consider

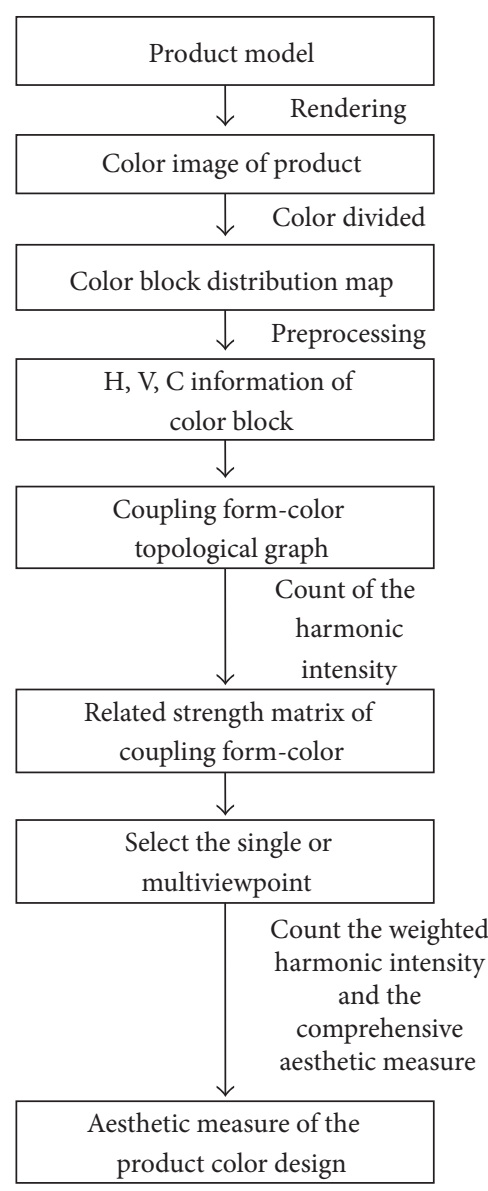

FIgURE 1: The flowchart of color aesthetic evaluation.

$\mathrm{O}_{i}=O h_{i}+O v_{i}+O c_{i}$. Any two colors of a color scheme form a color logarithm, $O_{i}$ is the reconcile factor of the color logarithm, and $O h_{i}, O v_{i}$, and $O c_{i}$ are the reconcile factor of hue, value, and chroma. $i=1,2, \ldots, n$ is the serial number of colors, $n$ is the number of all the color logarithms, $C_{m}$ is the number of colors, $C_{h}$ is the number of color logarithms with different hue, $C_{v}$ is the number of color logarithms with different value, and $C_{c}$ is the number of color logarithms with different chroma. The harmonic aesthetic feeling between the different colors can be concluded through the calculation of $M$.

However, the MS aesthetic measure model is based on the theory of two-dimensional harmonics [7] and it is not clear how to apply it to the harmony evaluation problem with more than three colors, such as (a), (b), and (c) shown in Figure 2; according to the theory of MS, there is no difference in the aesthetics of those color graphics, but there are large differences in human's visual feeling because of the different form and relative positions of those color graphics. The reason is that form is not evaluated in the aesthetic measure as a factor coupled with color.

Therefore, in this paper, form is taken into account in the aesthetic evaluation process as an important factor based on 


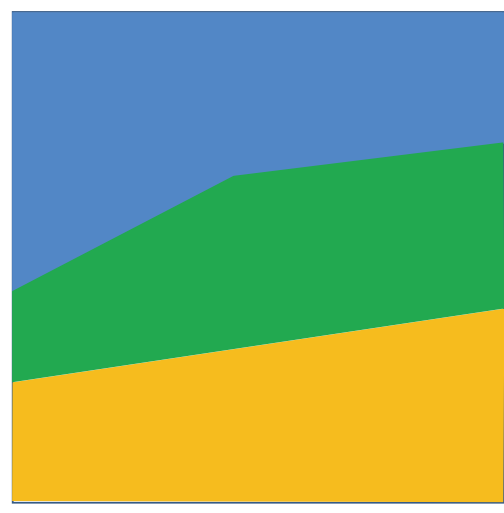

(a)

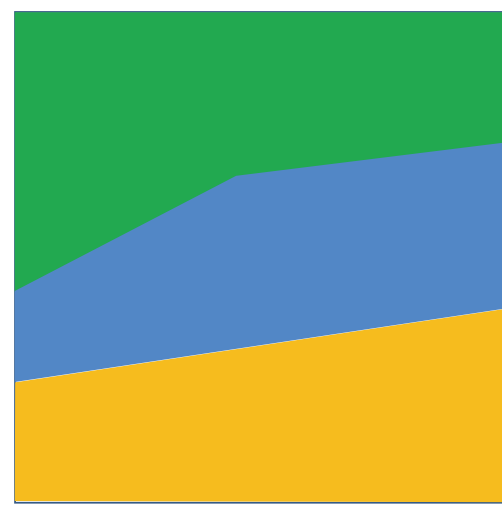

(b)

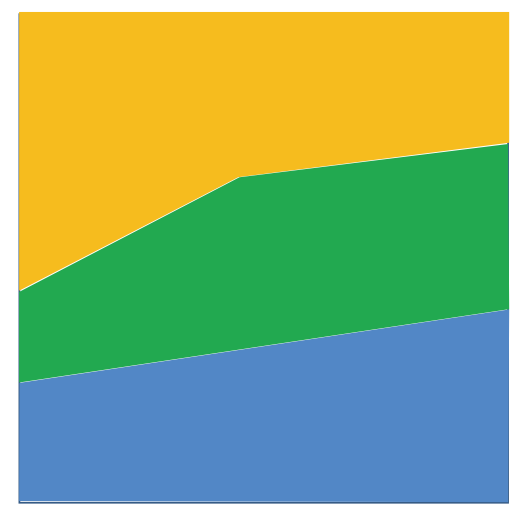

(c)

Figure 2: The difficult examples of MS color harmony theory.

the MS color harmony theory and we put forward a coupled form-color field aesthetic measure evaluation method according to the theory of psychophysical field.

2.2. The Concept of a Form-Color Field. Gestalt psychology is an important foundation to the cognitive and visual computing theory [12]. Koffka introduced the concept of "psycho-physical field" based on this theory and pointed out that the visual field has the characteristics of physical fields [13]. Physical field reveals the nature and laws of many psychological phenomena and provides an important foundation for the quantitative research of image.

In this paper, we introduce the concept of color block and form-color field based on the theory of psychophysical field.

Definition 1. A color block is an independent area with a certain shape which is composed of one color. For example, A, $\mathrm{B}, \mathrm{C}, \mathrm{D}$, and $\mathrm{E}$ are five color blocks in the color image shown in Figure 3.

Definition 2. Form-color field is the mental image where an objective image (including the form and color) takes shape in the visual cortex of human's brain $[14,15]$.

2.3. Topological Graph of Form. Due to the variations of the spatial relations between color blocks with the different composition of form, we could express the spatial relationship of color blocks using topological diagram.

Definition 3. Color block topological graph is an undirected graph with color blocks as the vertices and the adjacency relationships as edges. The topological graph of $\mathrm{A}, \mathrm{B}, \mathrm{C}, \mathrm{D}$, and $\mathrm{E}$ in Figure 3 is shown in Figure 4.

According to the combination of color blocks and their positional adjoining relationships, the topological relationships between color blocks could divide into direct connection relationship (DC) (such as $\mathrm{AB}, \mathrm{AD}, \mathrm{AE}$, and $\mathrm{BC}$ ) and indirect connections relationship (IC) (such as $\mathrm{AC}$, $\mathrm{BE}$, and $\mathrm{BD}$ ). The indirect connection relationship can be further divided into long indirect connection (such as $\mathrm{CD}$

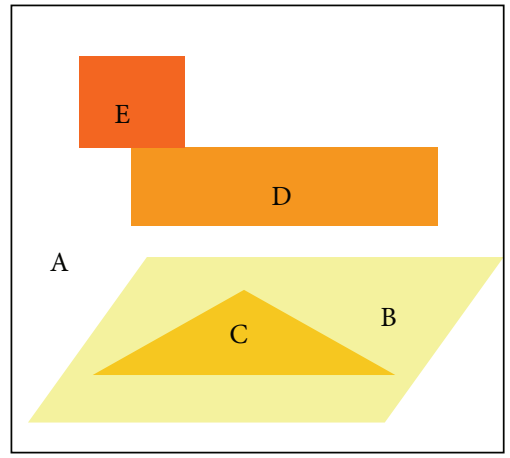

Figure 3: The color blocks in a color image.

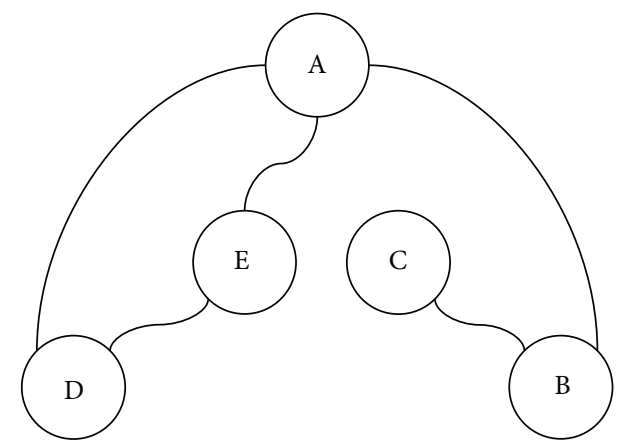

FIgURE 4: The form topological graph of color block.

and $\mathrm{CE}$ ) and short indirect connections (such as $\mathrm{AC}$ and $\mathrm{BE}$ ) according to the length of the path between the different color blocks (it is measured as the sum of pixel-distances between two color blocks).

2.4. Coupled Form-Color Topological Graph. After having clear spatial topological relations of each color block, this paper introduces the concept of harmonic intensity and develops a coupled form-color topological graph based on the topological graph of color blocks to elucidate the law of how the layout of forms affects the color harmony. 


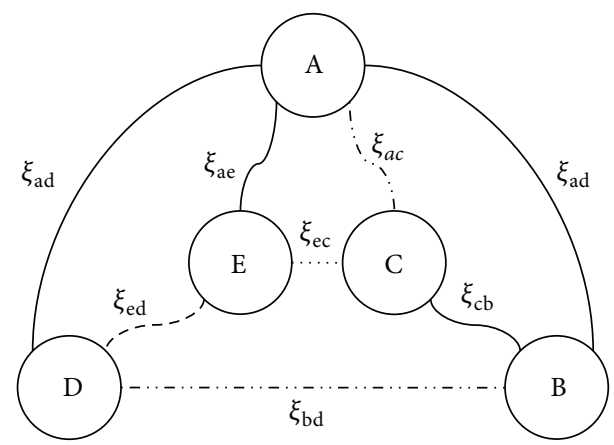

FIGURE 5: Coupled form-color topological graph.

Definition 4. Harmonic intensity $(\xi)$ refers to the impact measure of the harmonic relationship among the different form-color fields of a color image and it could be divided into four types that are strong, middle, weak, and nonharmonic. The corresponding fuzzy value could be set as 1.0, 0.6, 0.2 , and 0 .

Definition 5. Coupled form-color topological graph $G(V, E)$ is a harmonic relationships graph among different color blocks that is reestablished from the topological form graph of the color blocks by coupling (associating) each edge with a corresponding color harmonic intensity. The corresponding coupled form-color topological graph of Figure 3 could be shown as Figure 5.

Setting the vertices of a $G(V, E)$ as $V=\left\{v_{1}, v_{2}, \ldots, v_{p}\right\}$, $\xi_{i j}$ express the strength of a connection relationship between the color blocks $v_{i}$ and $v_{j}$, so, a related strength matrix $T(G)=$ $\left(\xi_{i j}\right)_{p \times p}$ can be established, shown as follows:

$$
T(G)=\begin{gathered}
v_{1} \\
v_{2} \\
\vdots \\
v_{i} \\
\vdots \\
v_{n}
\end{gathered}\left[\begin{array}{cccccc}
v_{1} & v_{2} & \cdots & v_{j} & \cdots & v_{n} \\
\xi_{11} & \xi_{12} & \cdots & \xi_{1 j} & \cdots & \xi_{1 n} \\
\xi_{21} & \xi_{22} & \cdots & \xi_{2 j} & \cdots & \xi_{2 n} \\
\vdots & \vdots & \cdots & \vdots & \cdots & \vdots \\
\xi_{i 1} & \xi_{i 2} & \cdots & \xi_{i 2} & \cdots & \xi_{i n} \\
\vdots & \vdots & \cdots & \vdots & \cdots & \vdots \\
\xi_{n 1} & \xi_{n 2} & \cdots & \xi_{n j} & \cdots & \xi_{n n}
\end{array}\right] .
$$

The calculation of the harmonic intensities in $T(G)$ is the key fundamental issue to establish the coupled formcolor topological graph and mathematical model [16]. It will directly affect the application of the coupled form-color topological graph in the color aesthetic intelligent evaluation system.

\section{The Algorithm to the Harmonic Intensity of Form-Color Field}

In this paper, a harmonic intensity evaluation model to the complex image is established based on the theory of psychophysical field and human's visual feeling [17] in the process of aesthetic evaluation. This model is useful to

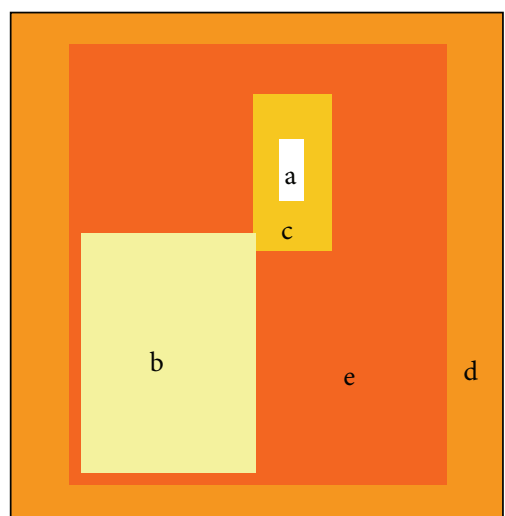

(A)

$\begin{array}{cccc}\text { Color block } & \text { Colors } & \text { Value of HV/C } & \text { Value of RGB } \\ \text { a } & \square & 10 / & 255,255,255 \\ \text { b } & \square & 0.6 \mathrm{GY} 9.7 / 6.1 & 255,255,155 \\ \text { c } & \square & 3.1 Y 8.3 / 12.4 & 255,204,9 \\ \text { d } & \square & 6 \text { YR7.1/13.7 } & 255,154,0 \\ \text { e } & \square & 9.5 R 5.9 / 17.2 & 255,91,0\end{array}$

(B)

FIGURE 6: The type of color block and its color information.

the expert system to realize the automatic evaluation of the computer intelligent system to color harmony aesthetic.

\subsection{The Measure Method of a Form-Color Field}

3.1.1. Measure of the Strength of Color Field. Based on the Munsell color calibration system, when we set the potential of gray in the psychophysical field as $E_{0}$, each color $E_{m}$ has a different potential $E_{i}$. So the expression of potential difference between colors $E_{m}$ and $E_{0}$ is

$$
E_{m}=E_{i}-E_{0} .
$$

$E_{i}$ can be described by three independent components $\left(x_{i}, y_{i}, z_{i}\right)$, which could be transformed from the corresponding HVC coordinates and the conversion formula is

$$
\begin{gathered}
x=c \cos h, \\
y=c \sin h, \\
z=v .
\end{gathered}
$$

Setting the origin coordinates of a gray point potential as $(0,0,0)$, the potential (energy), determined by the attributes of a color field, can be expressed as a Euclidean distance:

$$
E_{i}=\sqrt{x_{i}^{2}+y_{i}^{2}+z_{i}^{2}}
$$

So, the expression of the potential difference between the two color fields $E_{M}$ and $E_{N}$ is

$$
E_{m n}=\sqrt{\left(x_{i}-x_{j}\right)^{2}+\left(y_{i}-y_{j}\right)^{2}+\left(z_{i}-z_{j}\right)^{2}} \text {, }
$$




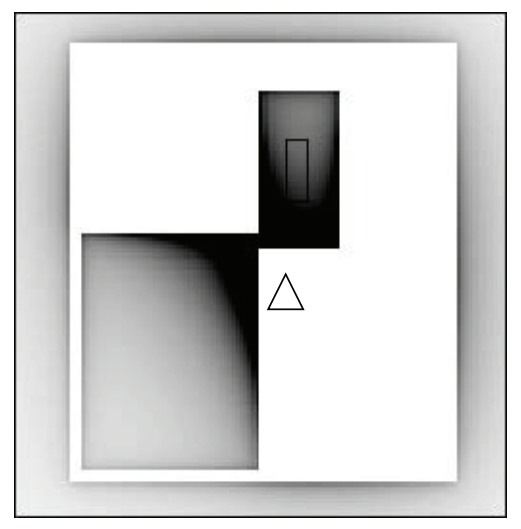

e1

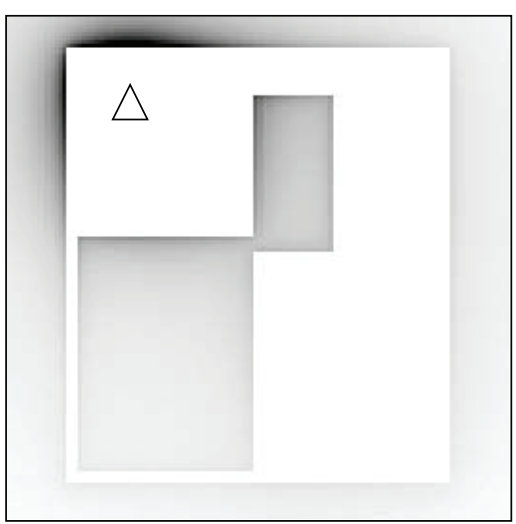

e2

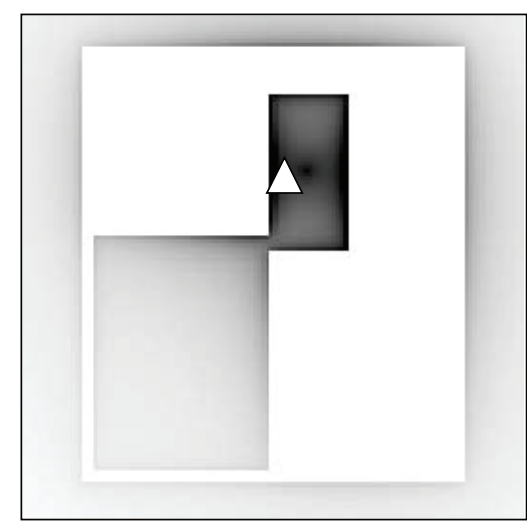

e3

FIgURE 7: The image of strength distribution of form-color field.

in which $E_{m n}$ is the potential difference of two form-color fields $\left(x_{i}, y_{i}, z_{i}\right)$ and $\left(x_{j}, y_{j}, z_{j}\right)$ expressing, respectively, the potential coordinates of two form-color fields.

3.1.2. Unit of Measure of a Visual Field. According to the visual characteristics of human [18], the relationship between the visual acuity of a point and the visual resolution or the minimum perceived threshold is

$$
\delta=\frac{60}{T_{\max }},
$$

in which $\delta$ is the visual resolution and its unit is second. $T_{\max }$ is the biggest visual acuity of the central fovea. So, the computational formula of the diameter of the minimum resolution cell of one visual field is

$$
\varepsilon=L \tan \delta \approx L \frac{\delta \pi}{180 \times 60 \times 60}=L \frac{\pi}{10800 T_{\max }},
$$

in which $L$ is the range of visibility and $\varepsilon$ is the diameter of the minimum sensor unit and its unit is $\mathrm{mm}$.

\subsection{Algorithm for Computing Harmonic Intensity of Form-Color Field}

3.2.1. The Calculation Principle of Harmonic Intensity. Suppose the area of a form-color field $F$ is $A$ and its average diameter size is $\varepsilon$; then the amount of perception of $F$ is represented as

$$
q=\frac{k A}{\varepsilon^{2}},
$$

in which $q$ is the number of perceived units of form-color field and $k$ is the correction factor of the amount of perception.

According to the psychophysical field theory, the harmonic intensity formula between form-color fields $F_{m}$ and $F_{n}$ is

$$
\xi_{m n}=k_{1} E_{m n} \frac{q_{m} q_{n}}{r_{m n}^{2}}
$$

Here $r_{m n}$ is the distance of visual perception between the color blocks $F_{m}$ and $F_{n}$, which could be related to the visual focus.

Because the color harmonic intensity range was set as $0 \sim 1$ in the actual observation, in order to evaluate the computational method and model by comparing the computed value with actual observation results, the harmonic intensity needs to be modified to its square roots prescription:

$$
\sqrt{\xi_{m n}}=\frac{\left(k_{1} E_{m n} q_{m} q_{n}\right)^{1 / 2}}{r_{m n}}
$$

3.2.2. The Example Analysis. Taking Figure 6(A) as an example, there are 5 color blocks, namely, a, b, c, d, and e, and their color information is shown in Figure 6(B).

After calculating the field strength of various colors according to our computational model, we obtained the field strength distributions of a single form-color field a, b, c, d, and $\mathrm{e}$ as shown in Figure 7, where " $\Delta$ " is the location of the visual focus. After that, we applied formulas (11) and (12) to calculate the harmonic intensity value $\xi$ between paired form-color fields as shown in Table 1. The table also shows the normalized values (normalized by the maximum harmonic intensity value under each viewpoint). Their distributions are given in Figure 8.

3.3. Experiment for Harmonic Intensity. The harmonic intensity of $\xi$ is varied with the color, form, size, and their topological relationships. Since people's psychological feeling has uncertainty, randomness, and fuzziness, it is easier to describe an actual harmonic intensity with a fuzzy variable [19]. In the paper we designed a psychophysical experiment to verify the results of the experiment and the fuzzy quantification calculation of the harmonic intensity of Figure 6 [20].

In this experiment we invited 40 industrial design undergraduate students and their ages range from 20 to 25 . They had been trained with color design for two years and with good color perception. With a D65 standard light as the source environment, the image was displayed on a $17^{\prime \prime}$ computer screen which had been adjusted to the color system. 


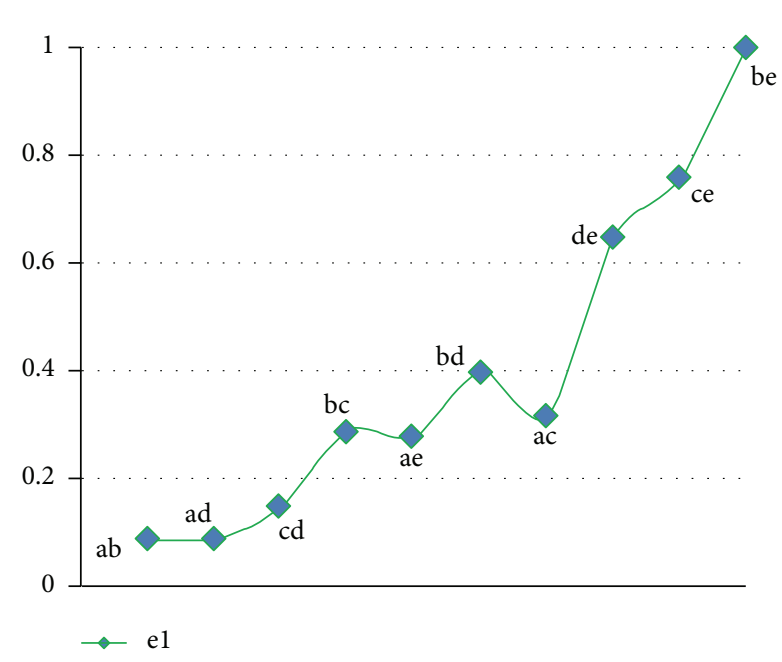

(a)

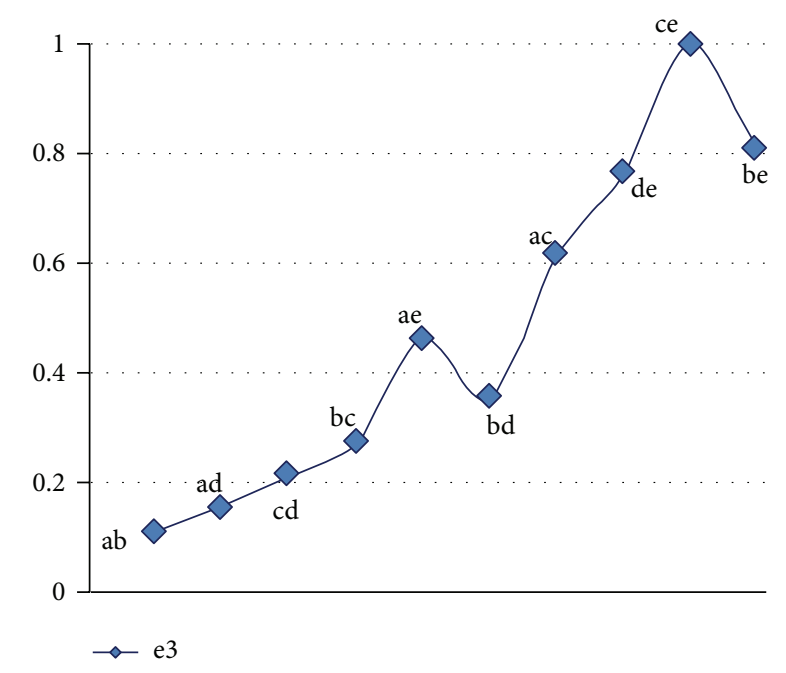

(c)

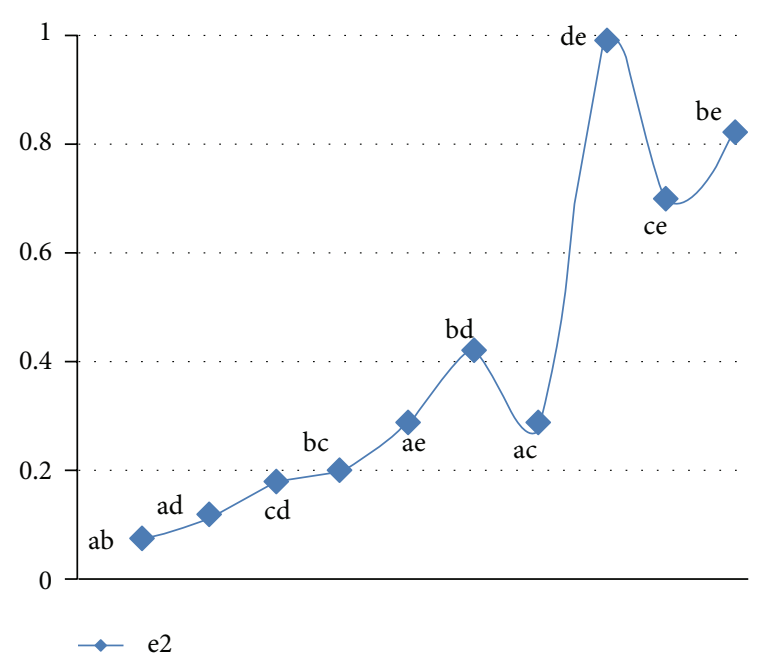

(b)

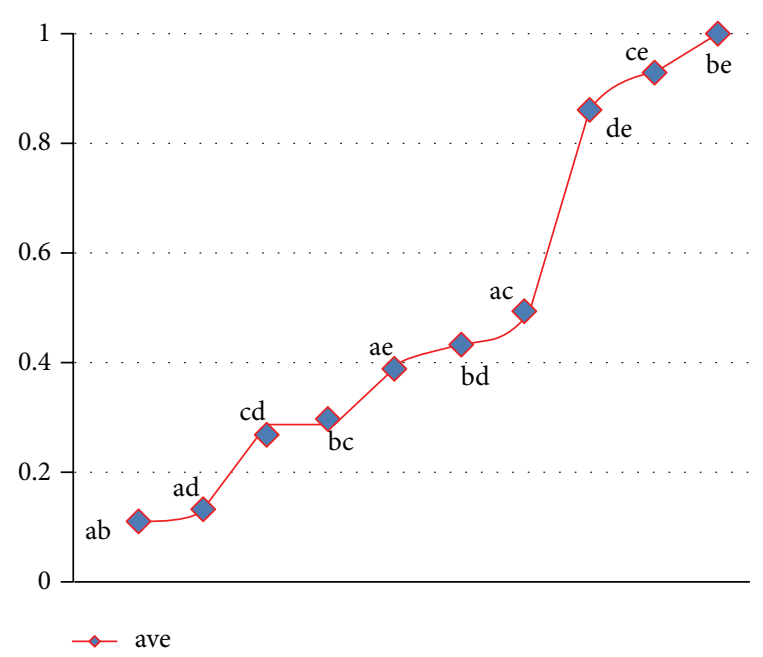

(d)

FIGURE 8: The distribution graph of color block.

Those students were required to sit 0.8 meters away from the screen and to adjust the seat to keep the line of sight vertical to the screen. Then, they were asked to observe the image quickly in Figure 6 within 1 minute and describe the harmonic intensity feeling of two color blocks with the language such as "strong, quite strong, intermediate, quite weak, weak, and nonharmonic relationship." From the descriptions, we established the corresponding fuzzy values as follows: $1.0 \sim 0.8,0.8 \sim 0.6,0.6 \sim 0.4,0.4 \sim 0.2$, and $0.2 \sim 0$. According to the language descriptions of the observers, we quantified the qualitative descriptions with the fuzzy quantitative values. The fuzzy quantitative results and the order of harmonic intensity of two color blocks are shown in Table 2.

Comparing Table 1 with Table 2, it is clear that the results of harmonic intensity order with different visual focus agree with the experiment results between two color fields. This result proved the effectiveness of the proposed algorithm.
However, there are some inconsistences with the harmonic intensity order of some form-color fields. The reason is that this example only calculated one visual focus, not considering the actual observation of the effect of visual attention. However, when we calculated the average of harmonic intensity of three different visual focuses, the order of the harmonic intensity of two color blocks is the same.

\section{Conclusion}

In this paper, we put forward the concepts of form topological graph, coupled form-color topological graph, and the harmonic intensity model based on the theory of psychophysical field and graph. We summarized the influence factors of color aesthetic measure such as color area, the distance, and the connection style of color blocks by psychophysical field experiment and established a harmonic intensity model. The model not only has important significance to reveal 
TABLE 1: The harmonic intensity of color blocks.

\begin{tabular}{|c|c|c|c|c|c|c|c|c|c|c|c|c|}
\hline \multirow{4}{*}{ Color block } & \multicolumn{12}{|c|}{ Visual focus } \\
\hline & \multicolumn{4}{|c|}{ el } & \multicolumn{2}{|l|}{$\mathrm{e} 2$} & \multicolumn{3}{|c|}{ e3 } & \multicolumn{3}{|c|}{ Average } \\
\hline & \multicolumn{12}{|c|}{ Harmonic intensity } \\
\hline & $\xi$ & $\sqrt{\bar{\xi}}$ & Normalized & $\xi$ & $\sqrt{\xi}$ & Normalized & $\xi$ & $\sqrt{\xi}$ & Normalized & $\xi$ & $\sqrt{\xi}$ & Normalized \\
\hline$\xi_{\mathrm{ab}}$ & 6.5782 & 2.56 & 0.087 & 2.0987 & 1.45 & 0.069 & 7.9441 & 2.82 & 0.11 & 5.5403 & 2.35 & 0.1 \\
\hline$\xi_{\mathrm{ad}}$ & 7.4501 & 2.73 & 0.09 & 5.3126 & 2.31 & 0.11 & 15.9642 & 4 & 0.15 & 9.5756 & 3.10 & 0.13 \\
\hline$\xi_{\mathrm{cd}}$ & 19.3815 & 4.4 & 0.15 & 14.1301 & 3.76 & 0.18 & 30.3586 & 5.51 & 0.21 & 37.9550 & 6.16 & 0.27 \\
\hline$\xi_{\mathrm{bc}}$ & 71.5843 & 8.46 & 0.29 & 17.2875 & 4.16 & 0.20 & 49.3929 & 7.03 & 0.27 & 46.0882 & 6.79 & 0.29 \\
\hline$\xi_{\mathrm{ae}}$ & 68.1364 & 8.25 & 0.28 & 34.3001 & 5.86 & 0.29 & 146.4624 & 12.1 & 0.46 & 82.9663 & 9.11 & 0.39 \\
\hline$\xi_{\mathrm{bd}}$ & 135.8970 & 11.66 & 0.4 & 76.8771 & 8.88 & 0.42 & 82.9019 & 9.11 & 0.35 & 98.5586 & 9.93 & 0.43 \\
\hline$\xi_{\mathrm{ac}}$ & 89.6409 & 9.20 & 0.32 & 37.5940 & 6.13 & 0.29 & 259.4902 & 16.11 & 0.61 & 128.9083 & 11.35 & 0.49 \\
\hline$\xi_{\mathrm{de}}$ & 363.265 & 19.05 & 0.65 & 443.45 & 21.06 & 1 & 395.78 & 19.89 & 0.76 & 401.4983 & 20.04 & 0.86 \\
\hline$\xi_{\mathrm{ce}}$ & 486.4252 & 22.05 & 0.76 & 219.891 & 14.83 & 0.7 & 694.08 & 26.34 & 1 & 466.799 & 21.61 & 0.93 \\
\hline$\xi_{\text {be }}$ & 849.344 & 29.14 & 1 & 306.186 & 17.5 & 0.83 & 456.3678 & 21.36 & 0.81 & 537.2995 & 23.18 & 1 \\
\hline
\end{tabular}

TABLE 2: The fuzzy quantitative results of the color harmonic intensity.

\begin{tabular}{|c|c|c|c|c|c|}
\hline \multirow{2}{*}{ Color block } & \multicolumn{5}{|c|}{ Intensity range } \\
\hline & $\xi=0.8-1.0$ & $\xi=0.6-0.8$ & $\xi=0.4-0.6$ & $\xi=0.2-0.4$ & $\xi=0-0.2$ \\
\hline be & $100 \%$ & 0 & 0 & 0 & 0 \\
\hline ce & $100 \%$ & 0 & 0 & 0 & 0 \\
\hline de & $97 \%$ & 0 & $3 \%$ & 0 & 0 \\
\hline ac & 0 & 0 & $100 \%$ & 0 & 0 \\
\hline bd & 0 & 0 & $86.7 \%$ & $13.3 \%$ & 0 \\
\hline ae & 0 & $6.7 \%$ & 0 & $93.3 \%$ & 0 \\
\hline bc & 0 & 0 & $6.7 \%$ & $90 \%$ & $3.3 \%$ \\
\hline $\mathrm{cd}$ & 0 & $13.3 \%$ & 0 & $86.7 \%$ & 0 \\
\hline ad & 0 & 0 & 0 & $6.7 \%$ & $93.3 \%$ \\
\hline$a b$ & 0 & 0 & 0 & 0 & $100 \%$ \\
\hline
\end{tabular}

the harmony mechanism of the form-color field but also has important academic values for artificial intelligence, cognitive science, perception science, and the development of psychology.

\section{Conflict of Interests}

The authors declare that there is no conflict of interests regarding the publication of this paper.

\section{Acknowledgments}

The project was supported by the 111 Project of China (B13044) and the Natural Science Basic Research Plan in Shaanxi Province of China (Program no. 2014JM7283).

\section{References}

[1] K. Sato and M. Oda, "The effect of color and shape on aesthetic evaluation of colored shape," in Proceedings of the 22nd IEEE International Symposium on Robot and Human Interactive Communication, pp. 478-483, Gyeongju, Republic of Korea, August 2013.

[2] J. Albers, Interaction of Color, Yale University, 1975.
[3] G. W. Granger, "Aesthetic measure applied to color harmony: an experimental test," The Journal of General Psychology, vol. 52, no. 2, pp. 205-212, 1955.

[4] M.-C. Chuang and L.-C. Ou, "Influence of a holistic color interval on color harmony," Color Research and Application, vol. 26, no. 1, pp. 29-39, 2001.

[5] H. Gurura, L. W. MacDonald, and H. Dalke, "Background: an essential factor in colour harmony," in Proceedings of the Interim Meeting of the International Color Association (AIC '04), pp. 239-242, 2004.

[6] L.-C. Ou, M. R. Luo, and G. Cui, "A colour design tool based on empirical studies," in Proceedings of the Design Research Society Conference, pp. 1-16, Sheffield, UK, 2008.

[7] L.-C. Ou and M. R. Luo, "A colour harmony model for twocolour combinations," Color Research and Application, vol. 31, no. 3, pp. 191-204, 2006.

[8] H.-C. Tsai and J.-R. Chou, "Automatic design support and image evaluation of two-coloured products using colour association and colour harmony scales and genetic algorithm," Computer Aided Design, vol. 39, no. 9, pp. 818-828, 2007.

[9] H.-C. Tsai, C.-Y. Hung, and F.-K. Hung, "Computer aided product color design with artificial intelligence," ComputerAided Design and Applications, vol. 4, no. 1-4, pp. 557-564, 2007.

[10] A. Kimura, Y. Wada, and K. Noguchi, "The effect of the similarity of affective meanings on harmony between shape and color," 
Bulletin of Japanese Society for the Science of Design, vol. 52, no. 6, pp. 1-8, 2006.

[11] P. Moon and D. E. Spencer, "Geometric formulation of classical color harmony," Journal of the Optical Society of America, vol. 34, pp. 46-59, 1944.

[12] L. Bo, History of Psychological Experiment, translated by J. Gao, Beijing Business Press, 1981.

[13] K. Koffka, Principle of Gestalt Psychology, translated by W. Li, Zhejiang Education Press, Hangzhou, China, 1997.

[14] H. Kolb, "How the retina works," The American Scientist, vol. 91, no. 1, pp. 28-55, 2003.

[15] H. Kolb, E. Fernandez, and R. Nelson, The organization of retina and visual system, 2005, http://webvision.med.utah.edu.

[16] D. Man, D. Wei, and Y. Chih-Chieh, "Product color design based on multi-emotion," Journal of Mechanical Science and Technology, vol. 27, no. 7, pp. 2079-2084, 2013.

[17] M. Hanada, "Analyses of color emotion for color pairs with independent component analysis and factor analysis," Color Research and Application, vol. 38, no. 4, pp. 297-308, 2013.

[18] M. Vlcěk, "Chebyshev polynomial approximation for activation sigmoid function," Neural Network World, vol. 22, no. 4, pp. 387393, 2012.

[19] L.-C. Ou, P. Chong, M. R. Luo, and C. Minchew, "Additivity of colour harmony," Color Research \& Application, vol. 36, no. 5, pp. 355-372, 2011.

[20] F. Szabó, P. Bodrogi, and J. Schanda, "Experimental modeling of colour harmony," Color Research and Application, vol. 35, no. 1 , pp. 34-49, 2010. 


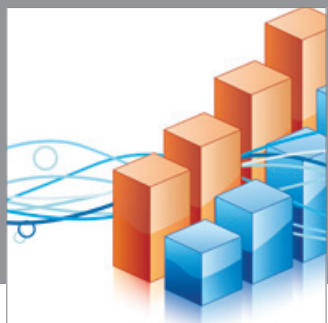

Advances in

Operations Research

mansans

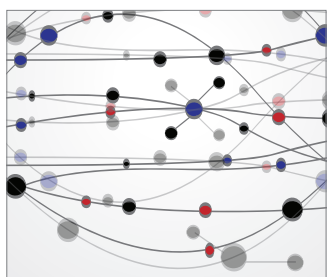

The Scientific World Journal
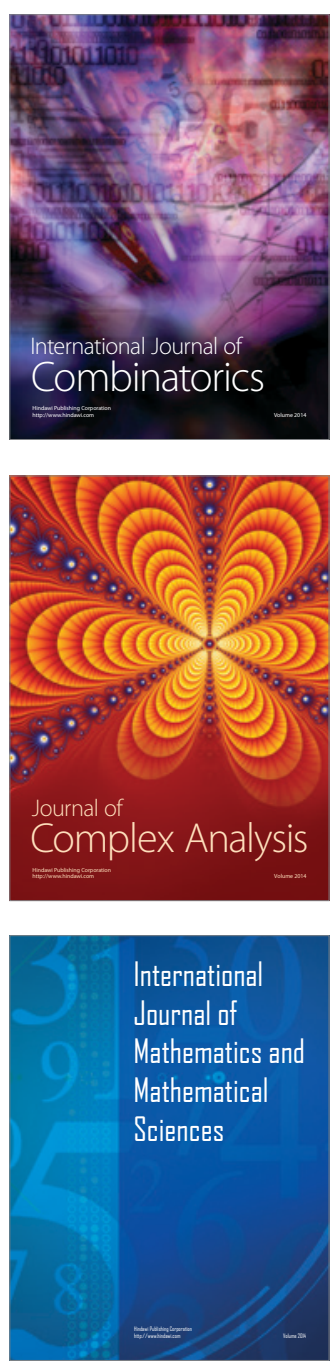
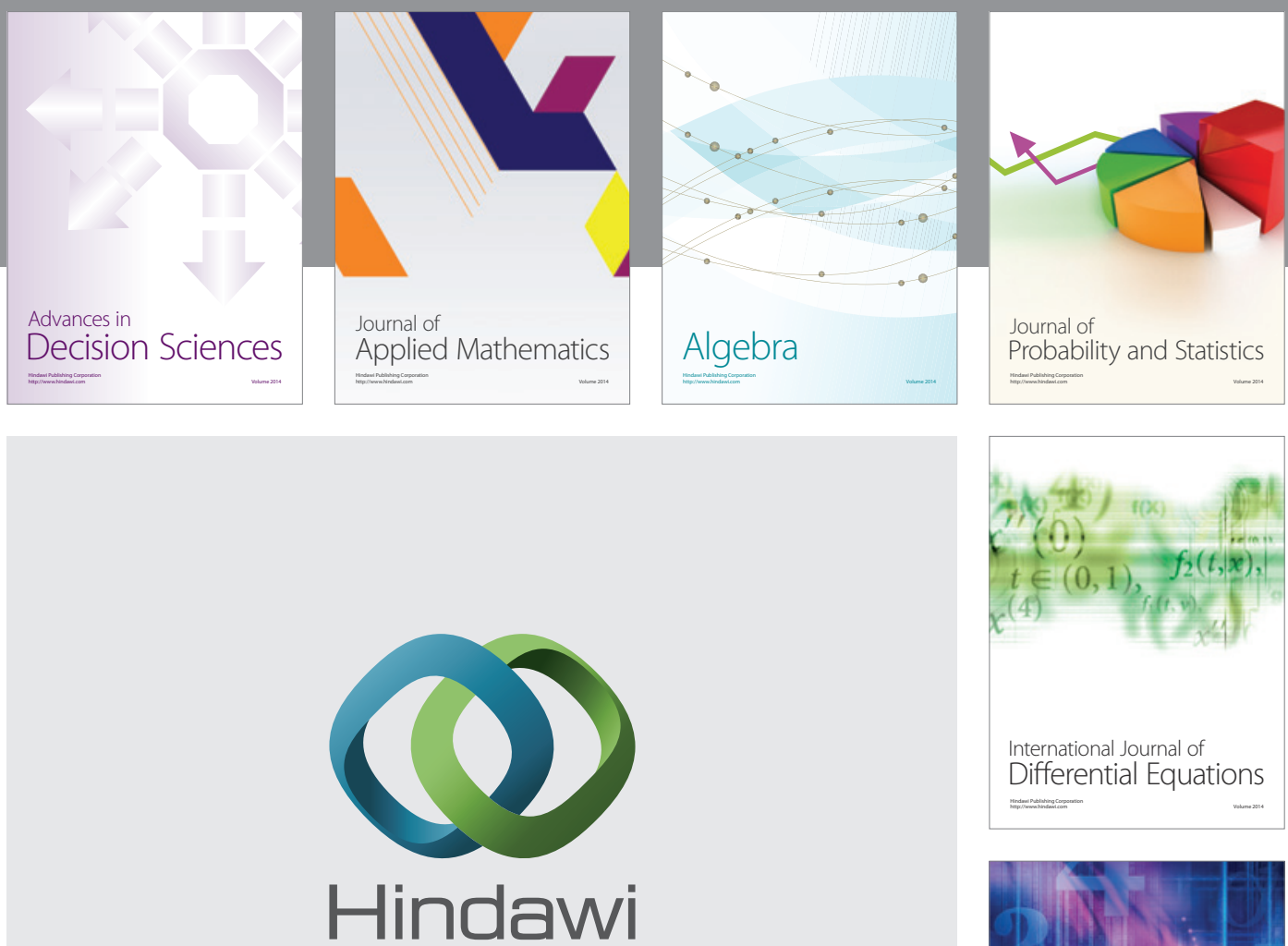

Submit your manuscripts at http://www.hindawi.com
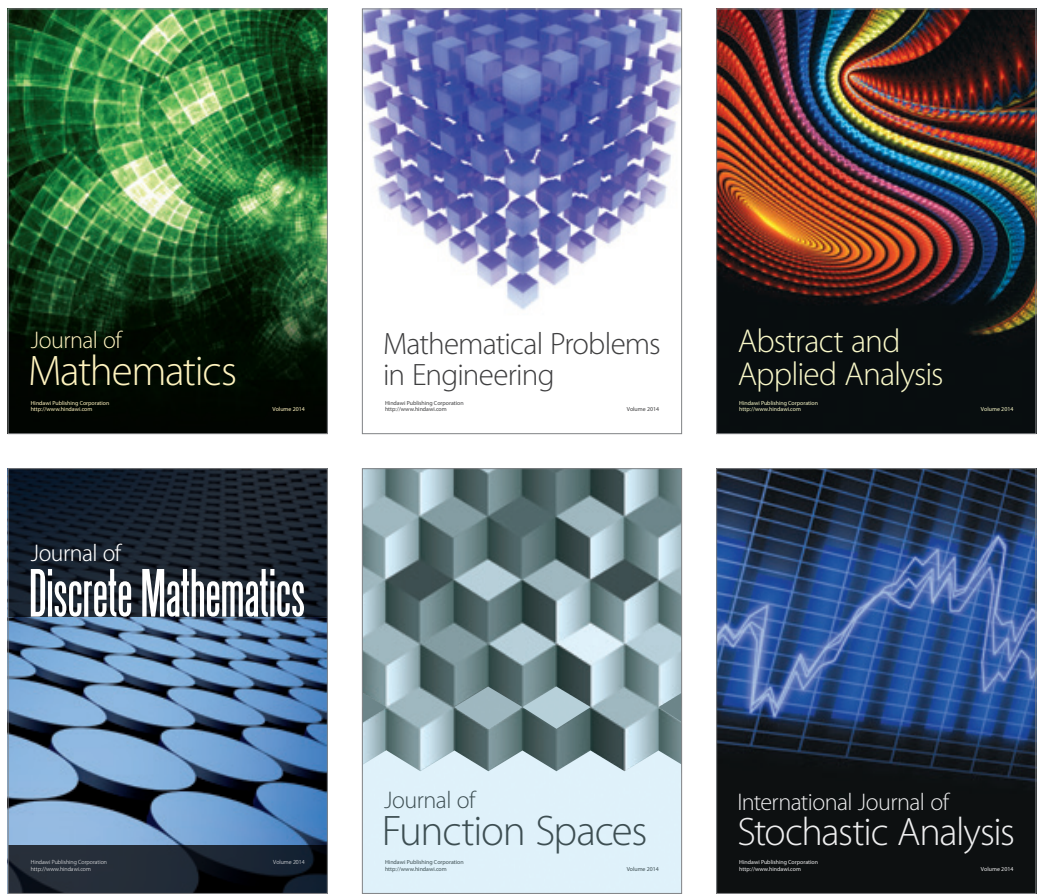

Journal of

Function Spaces

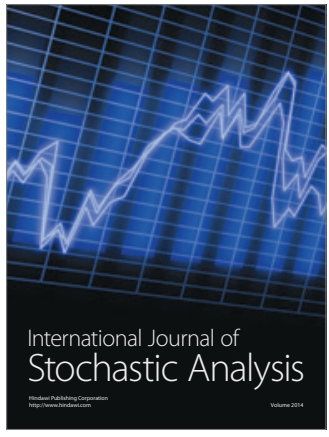

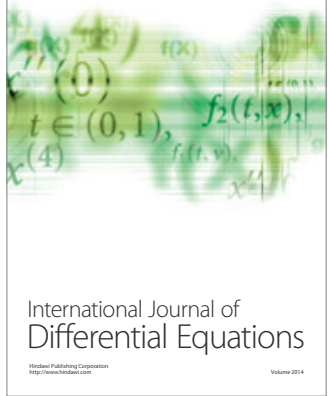
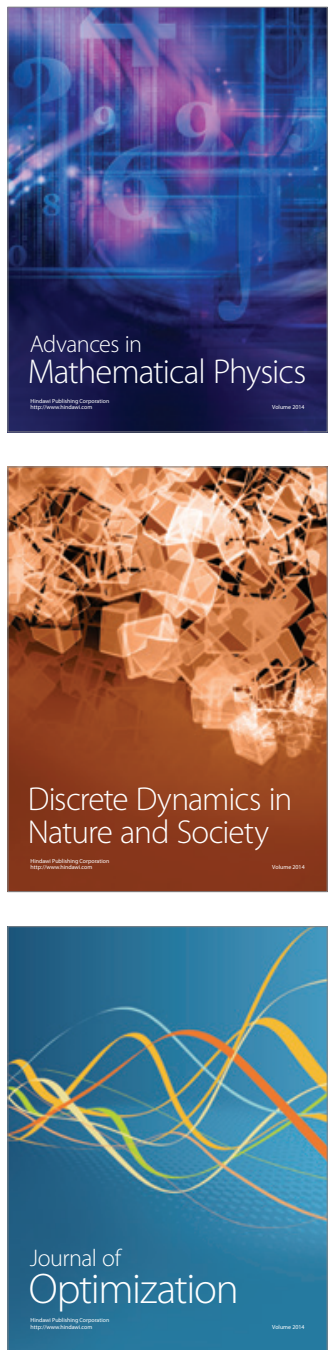